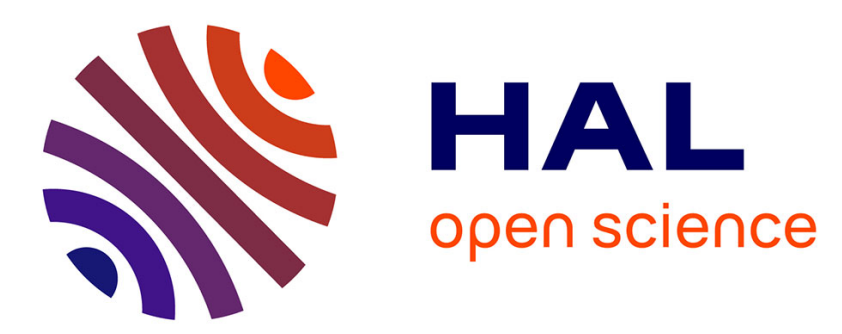

\title{
Observer and first-order low-pass filter based attitude estimation for rigid bodies subject to external acceleration
}

Tristan Bonargent, Tomas Menard, Eric Pigeon, Olivier Gehan

\section{To cite this version:}

Tristan Bonargent, Tomas Menard, Eric Pigeon, Olivier Gehan. Observer and first-order lowpass filter based attitude estimation for rigid bodies subject to external acceleration. 2019 IEEE 58th Conference on Decision and Control (CDC), Dec 2019, Nice, France. pp.629-634, 10.1109/CDC40024.2019.9030202 . hal-02276192

\section{HAL Id: hal-02276192 \\ https://hal.science/hal-02276192}

Submitted on 23 Mar 2020

HAL is a multi-disciplinary open access archive for the deposit and dissemination of scientific research documents, whether they are published or not. The documents may come from teaching and research institutions in France or abroad, or from public or private research centers.
L'archive ouverte pluridisciplinaire HAL, est destinée au dépôt et à la diffusion de documents scientifiques de niveau recherche, publiés ou non, émanant des établissements d'enseignement et de recherche français ou étrangers, des laboratoires publics ou privés. 


\title{
Observer and first-order low-pass filter based attitude estimation for rigid bodies subject to external acceleration
}

\author{
T. Bonargent* ${ }^{* \ddagger}$, T. Ménard*, E. Pigeon* and O. Gehan* \\ ${ }^{*}$ Normandie Univ, Unicaen, Ensicaen, LAC (EA 7478), 6 Bd Maréchal Juin, 14035 Caen Cedex, France \\ $\dagger$ KAD!, Espace Conquérant, 3 Place Jean Nouzille, 14000 Caen, France \\ ${ }_{\ddagger}^{\ddagger}$ Corresponding author, e-mail: tristan.bonargent@unicaen.fr
}

\begin{abstract}
In this paper, we consider the problem of estimating the attitude of a rigid body who is subject to non-negligible external acceleration, based on the measurements provided by a classical IMU unit: gyroscope, accelerometer and magnometer. Two algorithms are proposed, based on the combination of a low-pass filter in the fixed inertial frame and an observer. The stability of the proposed schemes are proved, based on a Lyapunov approach. Simulations are provided in order to illustrate the performances of the proposed observers.
\end{abstract}

\section{INTRODUCTION}

The estimation of attitude for a rigid body has been a great subject of interest for the last decades. An estimation of the attitude is indeed needed in many fields such as robotics [27], bio-logging [11], indoor positioning [30] or UAV [10]. Nowadays, the estimation of orientation is usually based on the measurement of two vectors in some body fixed frame whose reference value in an inertial fixed frame (usually earth fixed frame) is known, together with the angular velocity in body fixed frame. This usual configuration is mainly due to the development of low-cost systems equipped with MEMS inertial measurement unit, which contains an accelerometer, a magnometer and a gyroscope.

Many approaches have been developed for the reconstruction of the attitude, using these measurements. They can be divided into three main approaches (surveys can be found in [9] and [37] for example). The first category contains optimization based approaches and is usually referred to as the Wahba's problem [36], [7]. For this approach, no filtering is performed and only the measurements at time $t$ is used for the estimation of the orientation at time $t$. The second approach is based on stochastic filtering and contains different types of Kalman filters, such as EKF [20], [38], UKF [8] or particle filter [28]. Though providing interesting results, the main drawback is that convergence cannot be ensured in general and the tuning of the gains might not be easy. The third approach is based on nonlinear observers. The main advantage of this approach is that a large domain of convergence can be ensured and the tuning of the gains can be done through a systematic procedure. These observers can be designed directly on $S 0(3)$ [18], [5], [35], [22] or on $\mathbb{R}^{3 \times 3}$ (forgetting the underlying geometry structure) [1], [2], [25].
These approaches give satisfactory results when the considered body is subject to only low magnitude external acceleration. Indeed, it is usually assumed that the accelerometer provide a measurement of the gravity only, neglecting external acceleration. But this is generally not the case when medium to high acceleration are considered, which can occur when considering mobile robots such as UAV [13] or even when a smartphone is carried by a pedestrian [26].

In order to alleviate this problem, different solutions have been considered. Additional measurements have been considered, such as position provided by a GPS [13], [15], or velocity either in inertial frame [21], [12], [31] or in body fixed frame [4], [34], [14]. This allows to obtain an estimation of the external acceleration and thereby correcting the fact that the magnometer does not measure only the gravity in the body fixed frame. A second approach consists in using a model for the acceleration, but this can be used only for specific cases where such a model exists and is known such as in some robotic vehicles, for example for quadrotors [23], [24]. Another approach consists in using the fact that the norm of the accelerometer is known when there is no external acceleration, the difference $\mu$ between the norm of the measured acceleration and the gravity constant should actually be equal to zero. In [29], [32], [17], the accelerometer are discarded in the update phase if the value of $\mu$ is too high. Residual errors are used in [33], [19] to detect external acceleration. In [30], the update phase of the Kalman filter is only performed during periods considered as quasi static field, which is defined as a period of low variance of measurements.

No observer-based approach, with proof of stability, taking explicitly into account non negligible external acceleration without extra measurements or extra knowledge on the external acceleration model exists. An approach is then proposed here based on the assumption that the acceleration in the inertial frame can be decomposed into a low frequency component equal to the known constant gravity vector and an high frequency component equal to the external acceleration. It should be noted that this assumption is not true for the measured acceleration, since the measurements are done in 
the body fixed frame, which means that one cannot directly apply a low pass filter to the measured acceleration unless the angular velocity is very low. The proposed attitude estimator is composed of a first order low pass filter together with an observer. Furthermore the considered estimator forgets the underlying geometry of $S O(3)$, which means that the estimates belong to $\mathbb{R}^{3 \times 3}$. The drawback is that the dimension of the observer increases, but the advantage is that there is no topological limitation, unwinding phenomena or singularities for achieving global stability due the structure of $S O(3)$ [3], [6]. Two observers are proposed here. A first observer is based on a coordinate transformation which allows to obtain a linear error system, but the computation of the observer gain implies computing the inverse of a time-varying matrix. A second observer is then proposed with a different gain, which does not involve computing the inverse of this matrix.

The paper is organized as follows. The model used to design the observer is depicted in section II, together with some notations. Section III contains the proposed observers. The considered low-pass filter is given in subsection III-A. The two observers are presented in subsections III-B and III-C respectively. Some simulations are provided in section IV in order to illustrate the performances of the proposed approach. Finally, section V concludes the paper.

\section{PReliminaries}

\section{A. Notations}

Throughout the paper, a block diagonal matrix is represented as $\operatorname{diag}\left(A_{1}, \ldots, A_{n}\right)$. The identity matrix and the square zero matrix, of dimension $n \in \mathbb{N}$, are respectively denoted $I_{n}$ and $0_{n}$. For $x, y \in \mathbb{R}^{3}, x \times y$ represents the cross product. For a vector $x=\left(\begin{array}{lll}x_{1} & x_{2} & x_{3}\end{array}\right)^{T} \in \mathbb{R}^{3}, x_{\times} \in \mathbb{R}^{3 \times 3}$ is the associated skew-symmetric matrix, that is

$$
x_{\times}=\left(\begin{array}{ccc}
0 & -x_{3} & x_{2} \\
x_{3} & 0 & -x_{1} \\
-x_{2} & x_{1} & 0
\end{array}\right)
$$

The lower and upper eigenvalues of a matrix $M$ are denoted $\lambda_{\min }(M)$ and $\lambda_{\max }(M)$ respectively.

\section{B. Physical model}

Consider an inertial reference frame $\{I\}$ and a body fixed frame $\{B\}$. The rotation matrix from $\{B\}$ to $\{I\}$, of the considered moving body, is denoted $R(t) \in S O(3)$ and verifies the following differential equation

$$
\dot{R}(t)=R(t)(\omega(t))_{\times}, \quad t \geq 0
$$

where $\omega(t) \in \mathbb{R}^{3}$ is the angular velocity of the moving body in the frame $\{B\}$.

One assumes that the rate gyro measurements are available without bias. In addition to the rate gyro, one has access to the measurements of an accelerometer and a magnometer. The output $a$ of the accelerometer is in the following form

$$
a(t)=R^{T}(t)\left(a_{e}(t)-g_{0}\right)
$$

where $g_{0}=\left(\begin{array}{lll}0 & 0 & G\end{array}\right)$, with $G \approx 9.81$ the gravitational constant and $a_{e}(t)$ is the external acceleration, that is all other accelerations applied to the body.

The output $m$ of the magnometer is in the form

$$
m(t)=R^{T}(t) m_{I}
$$

where $m_{I}$ is the earth magnetic field (expressed in the inertial frame) and is constant.

In the remainder of the paper the following assumption is supposed to hold true.

Assumption 1. The vectors $a(t)$ and $m(t)$ are non-collinear for all $t \geq 0$, more precisely, there exists $\alpha>0$ such that $\|a(t) \times m(t)\| \geq \alpha, \forall t \geq 0$.

In a more general way, one assumes that 3 independent vectors $v_{i}(t) \in \mathbb{R}^{3}$ are measured in the body fixed frame, and are in the following form

$$
v_{i}(t)=R^{T}(t)\left(b_{i}+p_{i}(t)\right)
$$

where $b_{i} \in \mathbb{R}^{3}$ is a known constant reference vector and $p_{i}(t) \in \mathbb{R}^{3}$ is a time varying unknown perturbation. This is not restrictive since one can take $a \times m$ for the third vector, in order to obtain three linearly independent vectors, when only accelerometer and magnometer measurements are available. The perturbations $p_{i}, i=1,2,3$, and their derivative will be supposed to be bounded in the following.

Assumption 2. There exist $\gamma_{1}, \gamma_{2}>0$ such that $\left\|p_{i}(t)\right\| \leq \gamma_{1}$ and $\left\|\dot{p}_{i}(t)\right\| \leq \gamma_{2}$ for $i=1,2,3$ and all $t \geq 0$.

\section{Design model}

Since the observer is designed directly on $\mathbb{R}^{3 \times 3}$, forgetting the $S O(3)$ structure, one now writes the rotation matrix $R$ in vector form. Similarly as in [1], denoting

$$
R(t)=\left(z_{1}(t) \quad z_{2}(t) \quad z_{3}(t)\right)^{T}, z_{i}(t) \in \mathbb{R}^{3}, i=1,2,3
$$

one can write $R(t)$ as a vector, as follows

$$
x_{2}(t)=\left(z_{1}^{T}(t) \quad z_{2}^{T}(t) \quad z_{3}^{T}(t)\right)^{T} \in \mathbb{R}^{9}
$$

It is then straightforward to show that

$$
\dot{x}_{2}(t)=-S_{3}(\omega(t)) x_{2}(t)
$$

where

$$
S_{3}(x)=\operatorname{diag}\left(x_{\times}, x_{\times}, x_{\times}\right) \in \mathbb{R}^{9 \times 9}
$$

Following equation (5), the 3 measurements can be written in a general way as follows

$$
b_{i}+p_{i}(t)=W_{i}(t) x_{2}(t), \quad i=1,2,3
$$

with

$$
W_{i}(t)=\left(\begin{array}{ccc}
v_{i}^{T}(t) & 0_{1 \times 3} & 0_{1 \times 3} \\
0_{1 \times 3} & v_{i}^{T}(t) & 0_{1 \times 3} \\
0_{1 \times 3} & 0_{1 \times 3} & v_{i}^{T}(t)
\end{array}\right) \in \mathbb{R}^{3 \times 9}
$$

One then obtains, in compact form, the relation

$$
B+P(t)=W(t) x_{2}(t)
$$

with $B=\left(\begin{array}{lll}b_{1}^{T} & b_{2}^{T} & b_{3}^{T}\end{array}\right)^{T} \in \mathbb{R}^{9}$,

$P(t)=\left(\begin{array}{lll}p_{1}^{T}(t) & p_{2}^{T}(t) & p_{3}^{T}(t)\end{array}\right)^{T} \in \mathbb{R}^{9}$ and $W(t)=\left(\begin{array}{lll}W_{1}^{T}(t) & W_{2}^{T}(t) & W_{3}^{T}(t)\end{array}\right)^{T} \in \mathbb{R}^{9 \times 9}$

It should be noted that by assumptions 1 and 2, the matrix $W(t)$ is non singular and bounded for all $t \geq 0$, more 
precisely, one can further say that there exist $\nu_{1}, \nu_{2}>0$ such that

$$
\nu_{1} I_{9} \leq W(t) \leq \nu_{2} I_{9}, \quad \forall t \geq 0
$$

\section{OBSERVER DESIGN}

\section{A. Low-pass filter design}

One considers the linear time-varying system (9)-(13):

$$
\begin{aligned}
\dot{x}_{2}(t) & =-S_{3}(\omega(t)) x_{2}(t) \\
B+P(t) & =W(t) x_{2}(t)
\end{aligned}
$$

The aim is to reconstruct $x_{2}$, but the problem is that the output $B+P(t)$ is unknown since only $B$ is known. The idea is based on the assumption that the perturbations are not low frequency, then the output of the following augmented system

$$
\begin{aligned}
\dot{x}_{1}(t) & =\frac{1}{\tau}\left(-x_{1}(t)+W(t) x_{2}(t)\right) \\
\dot{x}_{2}(t) & =-S_{3}(\omega(t)) x_{2}(t) \\
y(t) & =x_{1}(t)
\end{aligned}
$$

is approximately equal to $B$, indeed $P(t)$ is filtered out by the first stage of system (17)-(18), which is simply a first order low pass filter, with time constant $\tau>0$, whose transfer function is given by $G(s)=\frac{1}{\tau s+1}$.

It should be noted that the augmented system (17)-(18)(19) is uniformly observable since the matrix $W(t)$ is non singular for all $t \geq 0$ by assumption 1 . Then the design of an observer is possible.

\section{B. First observer}

The first proposed observer is given by

$$
\begin{aligned}
& \dot{\hat{x}}_{1}(t)=\frac{1}{\tau}\left(-\hat{x}_{1}(t)+W(t) \hat{x}_{2}(t)\right)+k_{1}\left(B-\hat{x}_{1}(t)\right) \\
& \dot{\hat{x}}_{2}(t)=-S_{3}(\omega(t)) \hat{x}_{2}(t)+k_{2} W^{-1}(t)\left(B-\hat{x}_{1}(t)\right)
\end{aligned}
$$

where $K=\left(\begin{array}{ll}k_{1} & k_{2}\end{array}\right)^{T} \in \mathbb{R}^{2}$ is the gain of the observer and is chosen in such a way that the matrix

is Hurwitz, where

$$
\bar{A} \triangleq A-K C=\left(\begin{array}{cc}
-\frac{1}{\tau}-k_{1} & \frac{1}{\tau} \\
-k_{2} & 0
\end{array}\right)
$$

$$
A=\left(\begin{array}{cc}
-\frac{1}{\tau} & \frac{1}{\tau} \\
0 & 0
\end{array}\right), \quad C=\left(\begin{array}{ll}
1 & 0
\end{array}\right)
$$

One has the following convergence result in the perturbation free case.

Theorem 1. Assume that $P(t) \equiv 0$ and that the gains $k_{1}, k_{2}$ are chosen such that the matrix $\bar{A}$ is Hurwitz, then the state of observer (20) converges exponentially toward the state of system (17)-(18).

Proof. First notice that in the perturbation free case, one has $W(t) x_{2}(t)=B$, for all $t \geq 0$, then $\varepsilon(t)=x_{1}(t)-B$ converges exponentially to zero, since the first-order lowpass filter $G(s)=\frac{1}{\tau s+1}$ has a static gain equal to 1 . Thus, there exists $\alpha, \beta>0$ such that $\|\varepsilon(t)\|_{T} \leq \beta e^{-\alpha t}$.

Consider the error state $e=\left(\begin{array}{ll}e_{1}^{T} & e_{2}^{T}\end{array}\right)^{T}$, with $e_{1}=\left(x_{1}-\hat{x}_{1}\right)$,
$e_{2}=W \tilde{x}_{2}$ and $\tilde{x}_{2}=\left(x_{2}-\hat{x}_{2}\right)$. Since $P(t) \equiv 0$, one can show that

$$
\dot{W}(t)=W(t) S_{3}(\omega(t))
$$

And one can obtain that

$$
\dot{e}(t)=\bar{A} e(t)+K \varepsilon(t)
$$

The error signal $e(t)$ is thus given by

$$
e(t)=e^{\bar{A} t} e(0)+\int_{0}^{t} e^{\bar{A}(t-s)} \varepsilon(s) d s
$$

since $\bar{A}$ is assumed to be Hurwitz, there exists $\gamma, \varphi>0$ such that $\left\|e^{\bar{A} t}\right\| \leq \varphi e^{-\gamma t}$ (one can further assume without loss of generality that $\gamma \neq \alpha$ ), and so

$$
\|e(t)\| \leq \varphi e^{-\gamma t}\|e(0)\|+\beta \varphi \frac{e^{-\alpha t}-e^{-\gamma t}}{\gamma-\alpha}
$$

This shows the exponential convergence to zero of $e_{1}(t)$ and $e_{2}(t)$. Since $W(t)$ verifies (14), $\tilde{x}_{2}(t)$ also converges exponentially to zero.

The next corollary provides a practical stability result in the case of bounded external accelerations.

Corollary 1. Assume that the external acceleration satisfies assumption 2 and that $k_{1}, k_{2}$ are chosen such that the matrix $\bar{A}$ is Hurwitz, then the estimation error of observer (20) $\tilde{x}=\left(\begin{array}{ll}\tilde{x}_{1}^{T} & \tilde{x}_{2}^{T}\end{array}\right)^{T}$, with $\tilde{x}_{1}=x_{1}-\hat{x}_{1}$ and $\tilde{x}_{2}=x_{2}-\hat{x}_{2}$ is ultimately bounded, that is, there exists a bound $K>0$ such that

$$
\lim \sup _{t \rightarrow+\infty}\|\tilde{x}(t)\| \leq K
$$

Proof. In the case where the perturbation $P(t)$ is non zero, the dynamics of $W(t) x_{2}(t)$ is given by

$$
\frac{d}{d t}\left(W(t) x_{2}(t)\right)=\dot{P}(t)
$$

and the output of the first order filter by $x_{1}(t)=B+\varepsilon(t)$. The signal $\varepsilon(t)$ is bounded since it corresponds to the sum of two signals, the first one is the filtered version of $P(t)$ by the first order filter and the second one is a signal converging exponentially to zero, which is due to the possibly incorrect initial condition when filtering $B$.

Let us denote $e(t)=\left(\begin{array}{ll}e_{1}^{T} & e_{2}^{T}\end{array}\right)^{T}$, with $e_{1}=\tilde{x}_{1}, e_{2}=W \tilde{x}_{2}$, then, one has

$$
\dot{e}(t)=\bar{A} e(t)+K \varepsilon(t)+D \dot{P}(t)
$$

with $D=\left[\begin{array}{ll}0_{9} & I_{9}\end{array}\right]^{T}$. It is direct to see that the error $e(t)$ is practically bounded since the matrix $\bar{A}$ is Hurwitz, and $\varepsilon(t)$ and $\dot{P}(t)$ are uniformly bounded. It directly follows that $\tilde{x}_{1}$ and $\tilde{x}_{2}$ are ultimately bounded because of (14).

Remark 1. Corollary 1 only provides practical convergence. Indeed, there are two sources of error for the reconstruction of the attitude, first the output of system (17)-(18) is not perfectly known, since one assumes that it is equal to $B$ in the observer, and the model of $W(t) x_{2}(t)$ is uncertain, due to the non zero derivative of $P(t)$. It is then not possible to obtain an arbitrarily small error. Nevertheless, the estimate given by the proposed observer is very satisfactory, as illustrated with the simulations in section IV. A more precise effect of these two sources of error shall be done in future works in 
order to determine the optimal gains, but is beyond the scope of this article, due to the limited space.

\section{Second observer}

The second proposed observer is given by

$$
\begin{aligned}
\dot{\hat{x}}_{1}(t)= & \frac{1}{\tau}\left(-\hat{x}_{1}(t)+W(t) \hat{x}_{2}(t)\right) \\
& \quad+k_{1} W(t) W^{T}(t)\left(B-\hat{x}_{1}(t)\right) \\
\dot{\hat{x}}_{2}(t)=- & S_{3}(\omega(t)) \hat{x}_{2}(t)+k_{2} W^{T}(t)\left(B-\hat{x}_{1}(t)\right)
\end{aligned}
$$

where the gains are chosen as $k_{1}>0$ and $k_{2}=\frac{k_{1}}{2}$.

One has the following convergence result in the perturbation free case.

Theorem 2. Assume that $P(t) \equiv 0$ and that $k_{1}>0$ and $k_{2}=$ $\frac{k_{1}}{2}$, then the state of observer (30) converges exponentially toward the state of system (17)-(18).

Proof. One denotes $e=\left(\begin{array}{ll}e_{1}^{T} & e_{2}^{T}\end{array}\right)^{T}, e_{1}=x_{1}-\hat{x}_{1}, e_{2}=$ $W(t) \tilde{x}_{2}$ and $\tilde{x}_{2}=x_{2}-\hat{x}_{2}$. The error equation is given by

$$
\begin{aligned}
& \dot{e}_{1}=-\frac{1}{\tau} e_{1}+\frac{1}{\tau} e_{2}-k_{1} W W^{T} e_{1}+k_{1} W W^{T} \varepsilon \\
& \dot{e}_{2}=-\frac{k_{1}}{2} W W^{T} e_{1}+\frac{k_{1}}{2} W W^{T} \varepsilon
\end{aligned}
$$

Consider the following candidate Lyapunov function

$$
V(e)=e^{T} M e, \quad M=\left(\begin{array}{cc}
\frac{1}{4} I_{9} & -\frac{1}{4} I_{9} \\
-\frac{1}{4} I_{9} & \frac{1}{2} I_{9}
\end{array}\right)
$$

This is a valid candidate Lyapunov function since $M$ is definite positive and thus there exists $\varrho_{1}, \varrho_{2}>0$ such that $\varrho_{1}\|e\| \leq \sqrt{V(e)} \leq \varrho_{2}\|e\|$.

One has

$$
\dot{V}(e) \leq-e^{T} N e+2 \sigma_{2} \sqrt{V(e)}\|\varepsilon\|
$$

where

$$
N=\left(\begin{array}{cc}
\frac{1}{2 \tau}+\frac{k_{1} \lambda_{1}}{4} & -\frac{1}{2 \tau} \\
-\frac{1}{2 \tau} & \frac{1}{2 \tau}
\end{array}\right)
$$

with $\lambda_{1}=\inf _{t \geq 0} \lambda_{\min }\left(W(t) W^{T}(t)\right)$,

$\lambda_{2}=\sup _{t \geq 0} \lambda_{\max }\left(W(t) W^{T}(t)\right)$ and $\sigma_{2}=\frac{k_{1} \lambda_{2}}{8 \rho_{1}}$. One can note that $\lambda_{1}>0$ because of inequality (14) and $\lambda_{2}<+\infty$ because the perturbation $P(t)$ is assumed to be uniformly bounded.

Furthermore, since $N$ is positive definite as soon as $k_{1} \lambda_{1}>$ 0 , there exists $\sigma_{1}>0$ such that

$$
\dot{V}(e) \leq-2 \sigma_{1} V(e)+2 \sigma_{2} \sqrt{V(e)}\|\varepsilon\|
$$

Then

$$
\frac{d}{d t}(\sqrt{V(e)}) \leq-\sigma_{1} \sqrt{V(e)}+\sigma_{2}\|\varepsilon\|
$$

Applying the comparison lemma (lemma 3.4 p.102 [16]) and following the same lines as in the end of the proof of Theorem 1 gives the result.

Corollary 2. Assume that the external acceleration satisfies assumption 2 and the gains are chosen as $k_{1}>0$ and $k_{2}=\frac{k_{1}}{2}$, then the estimation error of observer (30) $\tilde{x}=\left(\begin{array}{ll}\tilde{x}_{1}^{T} & \tilde{x}_{2}^{T}\end{array}\right)^{T}$, with $\tilde{x}_{1}=x_{1}-\hat{x}_{1}$ and $\tilde{x}_{2}=x_{2}-\hat{x}_{2}$ is ultimately bounded, that is, there exists a bound $K>0$ such that

$$
\lim \sup _{t \rightarrow+\infty}\|\tilde{x}(t)\| \leq K
$$

The proof of Corollary 2 combines the same ideas as the ones of corollary 1 and Theorem 2 and is then omitted.

\section{Simulations}

The behavior of the proposed observers are now illustrated through simulations.

For all the simulations, the rotation dynamic is given by equation (2) and the angular velocity is depicted on figure 1. The rotation is initialized in such a way that the Euler angles Yaw, Pitch, Roll are equal to $\left[\begin{array}{lll}0.4383 & 0.9902 & 1.0074\end{array}\right]$ at time $t=0$. The measurements given by the accelerometer and the magnometer are taken as:

$$
\begin{aligned}
a(t) & =R^{T}(t)\left(a_{e}(t)-g_{0}\right)+\varepsilon_{a}(t) \\
m(t) & =R^{T}(t) m_{I}+\varepsilon_{m}(t)
\end{aligned}
$$

where $a_{e}$ is the external acceleration and depends on the simulation, $\varepsilon_{a}$ and $\varepsilon_{m}$ are white noise with mean equal to 0 and standard deviation equal to 1 and 0.1 respectively, $g_{0}=$ $\left[\begin{array}{lll}0 & 0 & 9.81\end{array}\right]$ and $m_{I}=\left[\begin{array}{lll}0.434 & -0.0091 & 0.9008\end{array}\right]$.

The observers presented in this paper will be compared with the classical observer from [18] with explicit error formulation, without bias estimation, since no bias is considered here. The tuning parameters of the Mahony observer are taken as $k_{1}=k_{2}=k_{p}=1$. The two proposed observers are implemented with the same gains $k_{1}=1$ and $k_{2}=1 / 2$. The time constant of the low-pass filter is taken as $\tau=2 s$.

All the implemented observers are initialized at $\hat{R}(0)=I_{3}$. When external acceleration is considered, it starts only after time $t=10 \mathrm{~s}$ in order for the observers to converge before. Furthermore, the mean square error (MSE) will be computed in order to compare the different observers, but between $t=$ $10 \mathrm{~s}$ and $t=100 \mathrm{~s}$ (the end of the simulation), in order to take into account only the effect, on the error, of the external acceleration and not the transient behavior.

Three different simulations are considered here. First, the external acceleration is equal to zero, only noise on the measurements is considered. The Euler angles with their reconstructed version and the error $\tilde{R}=\|R-\hat{R}\|$ are reported on figure 2. All three observers perform quite well, even if the observers proposed in this paper filter the noise better, which has a direct effect on the estimation. It can be seen that the second proposed observer convergence is slower than the first one. This is due to the fact that the error dynamics depend on $W(t) W^{T}(t)$ and then can become slower if the eigenvalues of this matrix are low, while for the first observer the error dynamics are independent from $W(t)$. The MSE of observer 1 , observer 2 and the Mahony observer are respectively equal to $1.00^{\circ}, 2.47^{\circ}$ and $4.60^{\circ}$.

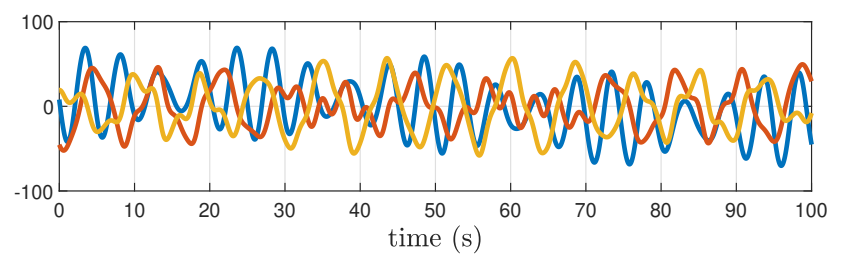

Fig. 1: Angular velocity $\omega(t)$ 


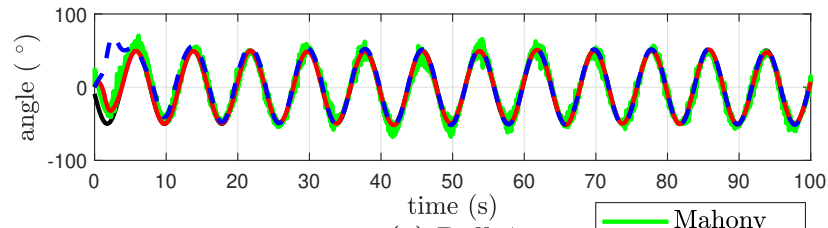

(a) Roll $\phi$

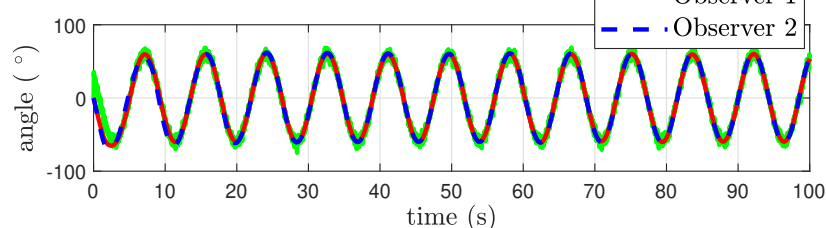

(b) Pitch $\theta$

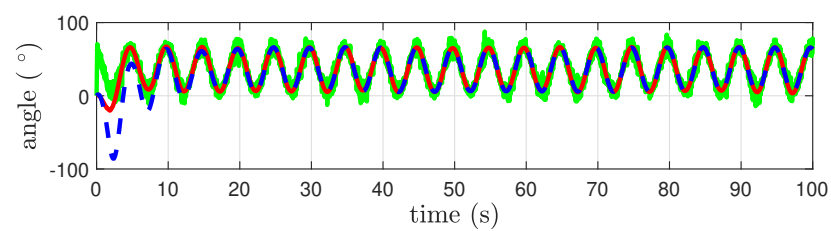

(c) Yaw $\psi$

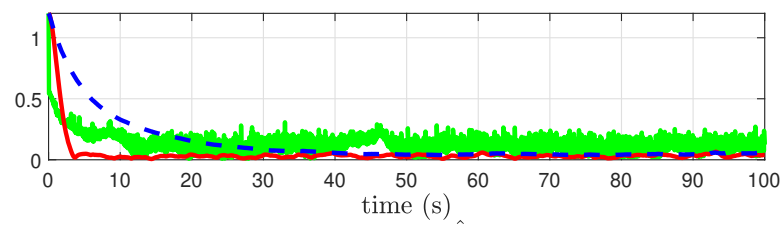

(d) $\|R-\hat{R}\|$

Fig. 2: Euler angles and error $\tilde{R}$ with no external acceleration A second simulation is considered with medium acceleration. The external acceleration applied on the rigid body is given on figure 3 with its filtered version. The external acceleration is well filtered, since its magnitude is divided by ten, which means that the time constant of the low-pass filter is correctly chosen. The Fourier transform of the applied external acceleration is depicted on figure 4. The norm of the error matrix $\|R-\hat{R}\|$ are reported on figure 5. The two proposed observer performed very well, while the impact of the external acceleration on the Mahony observer is quite high. The MSE of observer 1, observer 2 and the Mahony observer are respectively equal to $2.41^{\circ}, 3.80^{\circ}$ and $11.99^{\circ}$.

In the third simulation, the external acceleration is taken as twice the acceleration in the second simulation and is then quite high (the same order of magnitude as the gravitational constant). The reconstructed Euler angle and the error $\| R-$ $\hat{R} \|$ are given on figure 6. Observer 1 still performs very well, observer 2 estimations are pretty good, but the Mahony observer is completely off. The MSE of observer 1, observer 2 and the Mahony observer are respectively equal to $2.95^{\circ}$, $7.88^{\circ}$ and $33.53^{\circ}$.

\section{CONCLUSION}

We have presented two observers who provide an estimation of the attitude of a moving rigid body subject to external acceleration. The stability of these two observers are guaranteed through a Lyapunov approach. The good performances of the proposed approach have been illustrated through several simulations.

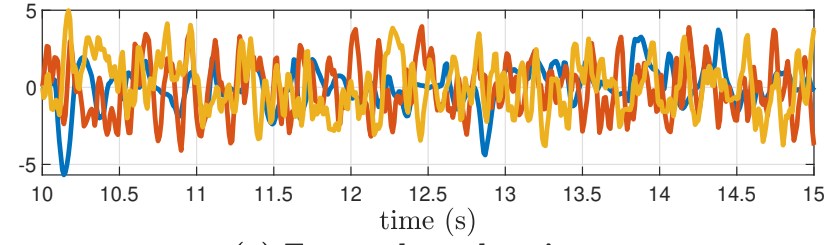

(a) External acceleration $a_{e}$

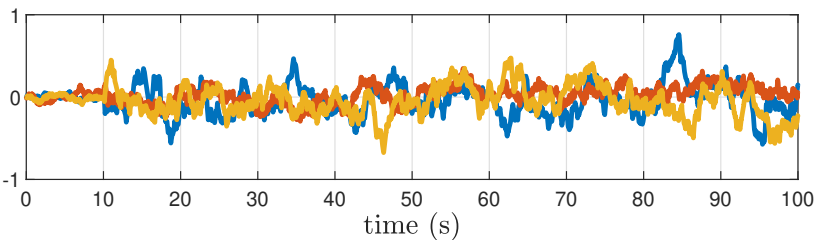

(b) Error between filtered external acceleration and $g_{0}$

Fig. 3: External acceleration and difference between the filtered acceleration and $g_{0}$ in the inertial frame for medium acceleration
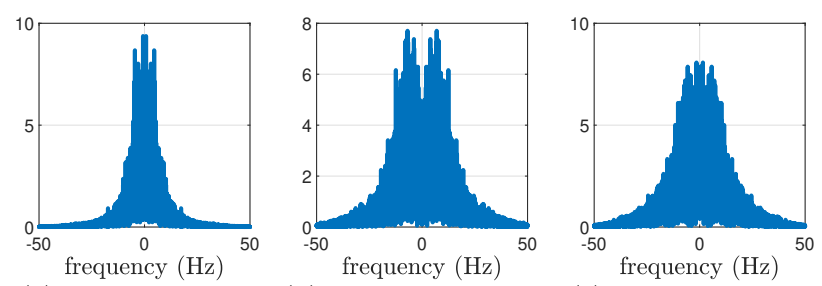

(a) First component

b) Second component

frequency $(\mathrm{Hz})$
(c) Third component

Fig. 4: Frequency representation of the external acceleration

The main drawback of the proposed approaches is the fact that the dimension of the proposed observer is higher than the classical $S O(3)$ observers, due to their geometry free structure. But the provided simulations show that the external acceleration are well filtered and the performances are very promising.

In future works, the authors will try to transform the observers so that their estimate evolve on $S O(3)$ in order to reduce the dimension of the proposed observers. Furthermore, a thorough analysis of the effect of the external acceleration on the estimation error would be interesting in order to tune the gains in an optimal way.

\section{REFERENCES}

[1] P. Batista, C. Silvestre, and P. Oliveira. Globally exponentially stable cascade observers for attitude estimation. Control Engineering Practice, 20(2):148-155, 2012.

[2] P. Batista, C. Silvestre, and P. Oliveira. Attitude and earth velocity estimation-part i: Globally exponentially stable observer. In 53rd IEEE Conference on Decision and Control, pages 121-126. IEEE, 2014.

[3] S.P. Bhat and D.S. Bernstein. A topological obstruction to continuous global stabilization of rotational motion and the unwinding phenomenon. Systems \& Control Letters, 39(1):63-70, 2000.

[4] S. Bonnabel, P. Martin, and P. Rouchon. Symmetry-preserving observers. arXiv preprint math/0612193, 2006.

[5] S. Bonnabel, P. Martin, and P. Rouchon. Symmetry-preserving observers. IEEE Transactions on Automatic Control, 53(11):2514-2526, 2008.

[6] N.A. Chaturvedi, A.K. Sanyal, and N.H. McClamroch. Rigid-body attitude control. IEEE control systems magazine, 31(3):30-51, 2011.

[7] D. Choukroun. Novel methods for attitude determination using vector observations. Technion-Israel Institute of Technology, Faculty of Aerospace Engineering, 2003. 


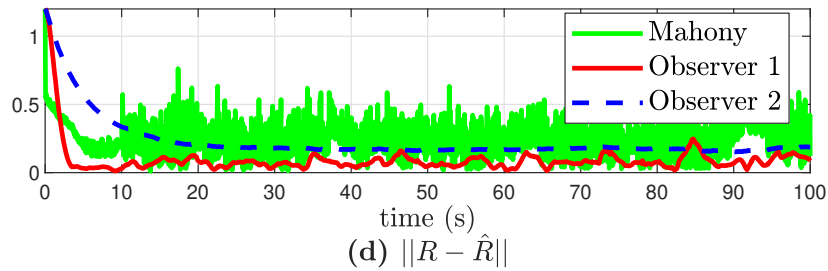

Fig. 5: Error $\tilde{R}$ with medium external acceleration

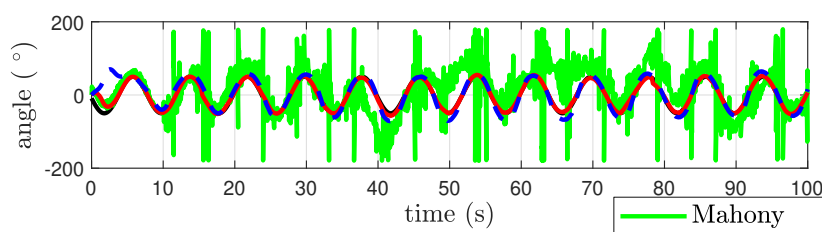

(a) Roll $\phi$

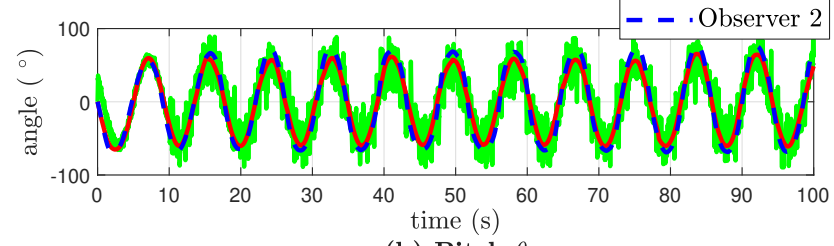

(b) Pitch $\theta$

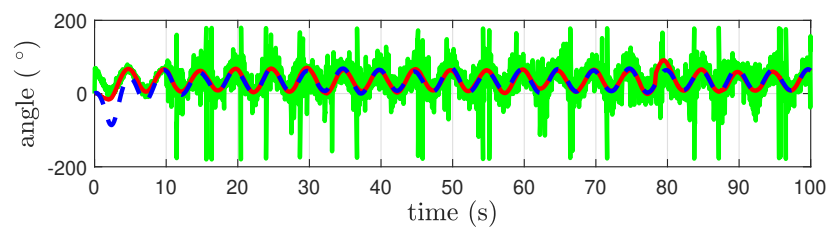

(c) Yaw $\psi$

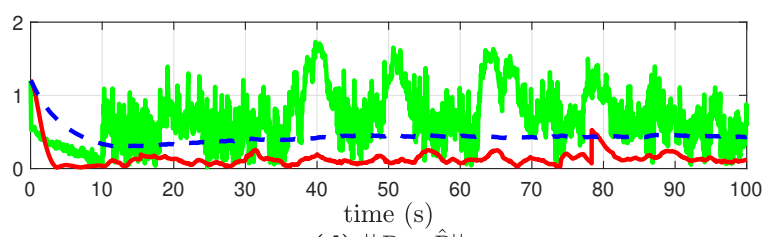

(d) $\|R-\hat{R}\|$

Fig. 6: Euler angles and error $\tilde{R}$ with high external acceleration

[8] J.L. Crassidis and F.L. Markley. Unscented filtering for spacecraft attitude estimation. Journal of guidance, control, and dynamics, 26(4):536-542, 2003.

[9] J.L. Crassidis, F.L. Markley, and Y. Cheng. Survey of nonlinear attitude estimation methods. Journal of guidance, control, and dynamics, 30(1):12-28, 2007.

[10] M. Euston, P. Coote, R. Mahony, J. Kim, and T. Hamel. A complementary filter for attitude estimation of a fixed-wing uav. In 2008 IEEE/RSJ International Conference on Intelligent Robots and Systems, pages 340-345. IEEE, 2008.

[11] H. Fourati, N. Manamanni, L. Afilal, and Y. Handrich. A nonlinear filtering approach for the attitude and dynamic body acceleration estimation based on inertial and magnetic sensors: Bio-logging application. IEEE Sensors Journal, 11(1):233-244, 2011.

[12] H.F. Grip, A. Saberi, and T.A. Johansen. Observers for interconnected nonlinear and linear systems. Automatica, 48(7):1339-1346, 2012

[13] M.D. Hua. Attitude estimation for accelerated vehicles using gps/ins measurements. Control Engineering Practice, 18(7):723-732, 2010.

[14] M.D. Hua, P. Martin, and T. Hamel. Stability analysis of velocityaided attitude observers for accelerated vehicles. Automatica, 63:11$15,2016$.

[15] T.A. Johansen, J.M. Hansen, and T.I. Fossen. Nonlinear observer for tightly integrated inertial navigation aided by pseudo-range measurements. Journal of Dynamic Systems, Measurement, and Control, 139(1):011007, 2017

[16] H.K. Khalil. Nonlinear systems. Prentice hall Upper Saddle River, third edition, 2002

[17] J.K. Lee, E.J. Park, and S.N. Robinovitch. Estimation of attitude and external acceleration using inertial sensor measurement during various dynamic conditions. IEEE transactions on instrumentation and measurement, 61(8):2262-2273, 2012.

[18] R. Mahony, T. Hamel, and J.M. Pflimlin. Nonlinear complementary filters on the special orthogonal group. IEEE Transactions on automatic control, 53(5):1203-1217, 2008.

[19] A. Makni, H. Fourati, and A.Y. Kibangou. Adaptive kalman filter for mems-imu based attitude estimation under external acceleration and parsimonious use of gyroscopes. In 2014 European Control Conference (ECC), pages 1379-1384. IEEE, 2014.

[20] J.L. Marins, X. Yun, E.R. Bachmann, R.B. McGhee, and M.J. Zyda. An extended kalman filter for quaternion-based orientation estimation using marg sensors. In Proceedings 2001 IEEE/RSJ International Conference on Intelligent Robots and Systems., volume 4, pages 20032011. IEEE, 2001.

[21] P. Martin and E. Salaün. An invariant observer for earth-velocityaided attitude heading reference systems. IFAC Proceedings Volumes, 41(2):9857-9864, 2008.

[22] P. Martin and E. Salaün. Design and implementation of a lowcost observer-based attitude and heading reference system. Control Engineering Practice, 18(7):712-722, 2010.

[23] P. Martin and E. Salaün. The true role of accelerometer feedback in quadrotor control. In 2010 IEEE International Conference on Robotics and Automation, pages 1623-1629. IEEE, 2010.

[24] P. Martin and I. Sarras. A semi-global model-based state observer for the quadrotor using only inertial measurements. In 2016 IEEE 55th Conference on Decision and Control (CDC), pages 7123-7128. IEEE, 2016.

[25] P. Martin and I. Sarras. A simple global observer for attitude and gyro biases. arXiv preprint arxiv:1604.03714, 2016.

[26] T. Michel, P. Genevès, H. Fourati, and N. Layaïda. On attitude estimation with smartphones. In 2017 IEEE International Conference on Pervasive Computing and Communications (PerCom), pages 267275. IEEE, 2017.

[27] L. Ojeda and J. Borenstein. Flexnav: Fuzzy logic expert rule-based position estimation for mobile robots on rugged terrain. In Proceedings 2002 IEEE International Conference on Robotics and Automation (Cat. No. 02CH37292), volume 1, pages 317-322. IEEE, 2002.

[28] Y. Oshman and A. Carmi. Attitude estimation from vector observations using a genetic-algorithm-embedded quaternion particle filter. Journal of Guidance, Control, and Dynamics, 29(4):879-891, 2006.

[29] H. Rehbinder and X. Hu. Drift-free attitude estimation for accelerated rigid bodies. Automatica, 40(4):653-659, 2004.

[30] V. Renaudin and C. Combettes. Magnetic, acceleration fields and gyroscope quaternion (magyq)-based attitude estimation with smartphone sensors for indoor pedestrian navigation. Sensors, 14(12):2286422890, 2014.

[31] A. Roberts and A. Tayebi. On the attitude estimation of accelerating rigid-bodies using gps and imu measurements. In 2011 50th IEEE Conference on Decision and Control and European Control Conference, pages 8088-8093. IEEE, 2011.

[32] A.M. Sabatini. Quaternion-based extended kalman filter for determining orientation by inertial and magnetic sensing. IEEE Transactions on Biomedical Engineering, 53(7):1346-1356, 2006.

[33] Y.S. Suh. Orientation estimation using a quaternion-based indirect kalman filter with adaptive estimation of external acceleration. IEEE Transactions on Instrumentation and Measurement, 59(12):3296-3305, 2010.

[34] G. Troni and L.L. Whitcomb. Preliminary experimental evaluation of a doppler-aided attitude estimator for improved doppler navigation of underwater vehicles. In 2013 IEEE International Conference on Robotics and Automation, pages 4134-4140. IEEE, 2013.

[35] J.F. Vasconcelos, C. Silvestre, and P. Oliveira. A nonlinear observer for rigid body attitude estimation using vector observations. IFAC Proceedings Volumes, 41(2):8599-8604, 2008

[36] G. Wahba. A least squares estimate of satellite attitude. SIAM review, 7(3):409-409, 1965.

[37] M. Zamani, J. Trumpf, and R. Mahony. Nonlinear attitude filtering: A comparison study. arXiv preprint arXiv: 1502.03990, 2015.

[38] R. Zhu, D. Sun, Z. Zhou, and D. Wang. A linear fusion algorithm for attitude determination using low cost mems-based sensors. Measurement, 40(3):322-328, 2007. 